\title{
Numerical simulation of a MEMS Pirani vacuum sensor
}

\author{
S. Quednau', H. F. Schlaak', F. Völklein ${ }^{2}$ \\ ${ }^{1}$ Technische Universität Darmstadt, Institute of Electromechanical Design, \\ Merckstraße 25, 64283 Darmstadt, Germany \\ s.quednau@emk.tu-darmstadt.de \\ ${ }^{2}$ RheinMain University of Applied Sciences, Institute for Microtechnologies, \\ Am Brückweg 26, 65428 Rüsselsheim, Germany
}

\begin{abstract}
This work presents a method to simulate the heat exchange characteristics of a MEMS Pirani vacuum sensor. The simulation couples the electro-thermal sensor system with the surrounding rarefied gas atmosphere. The heat exchange between the sensor and the surrounding gas is modelled by the exchange of boundary conditions between the solid and the gas (fluid) region. The dilution effects which occur under rarefied gas atmosphere are taken into account by an adapted slip boundary condition. The simulation has been carried out using a specially adapted solver which is implemented in OpenFOAM. The simulated results are in excellent agreement with the measured data of a MEMS Pirani vacuum sensor.
\end{abstract}

\section{Introduction}

Pirani vacuum sensors are widely used for vacuum pressure measurements ranging from $10^{-3}$ mbar to $10^{3}$ mbar [1]. Classical Pirani vacuum sensors usually consist of an electrically heated wire and a heat sink (see Fig. 1). The working principle of Pirani vacuum sensors is based on the pressure dependent heat loss of the wire.

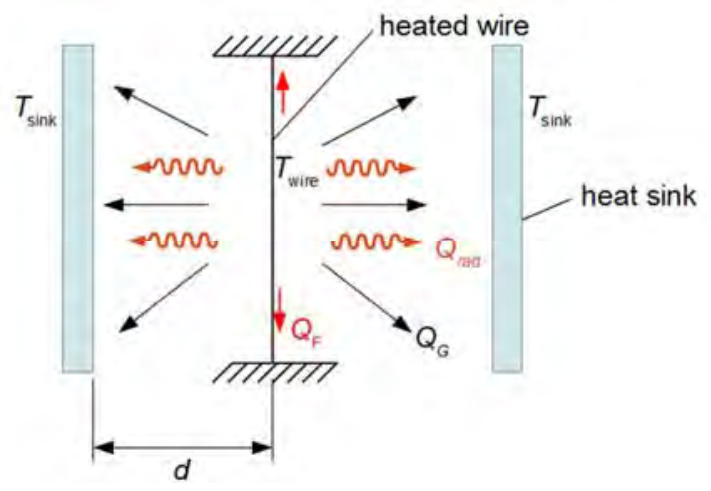

Fig. 1: Schematic view of a classical Pirani vacuum sensor. The wire is electrically heated and the pressure dependent heat loss is measured. The heat dissipates from the wire with three different thermal flows $Q_{\text {solid }}, Q_{\text {rad }}$ and $Q_{\text {gas }}$.

The electrically generated heat $P_{\mathrm{el}}=I \cdot R^{2}$ dissipates from the wire by three different mechanisms (see Fig. 1):

1. $Q_{\text {solid }}$ : Heat diffusion through the solid suspension of the heated wire

2. $Q_{\text {rad }}$ : Heat radiation of the hot wire
3. $Q_{\text {gas }}$ : Heat diffusion through the gas Only heat transport through the gas $Q_{\text {gas }}$ is pressure dependent. At lower pressures, fewer gas molecules collide with the heated wire. Therefore, at low pressures the heat dissipation from the wire is lower than at higher pressures. This results in a higher temperature of the heated wire to be be detected by the measurement of the wire's resistance.

\section{Analytical modeling of Pirani vacuum sen- sors}

The heat loss through the gas $Q_{\text {gas }}$ is proportional to the thermal conductivity $K(p)$ of the surrounding gas and it is proportional to the temperature difference between the hot wire and the heat sink:

$$
Q_{\text {gas }} \propto \kappa(p) \cdot\left(T_{\text {wire }}-T_{\text {sink }}\right)
$$

Due to gas dilution, the thermal conductivity $\kappa(\mathrm{p})$ becomes pressure dependent and can be modeled by eq. (2) [2]

$$
\kappa(p)=\frac{\kappa_{0}}{1+2 \frac{p_{0}}{p} \frac{\overline{l_{0}}}{d}\left(\frac{2-\sigma_{T}}{\sigma_{T}}\right)}
$$

where $p$ is the pressure, $\sigma_{T}$ is the thermal accommodation coefficient, $\kappa_{0}$ is the reference thermal conductivity, $p_{0}$ is the reference pressure and $\overline{l_{0}}$ is the mean free path at reference pressure.

In addition to pressure dependence the thermal conductivity depends on the gap distance $d$. 
Fig. 2 depicts the normalized thermal conductivity for three different gap distances $d$ (eq. (2)). The operating range of the sensor is surrounded by two saturated regions. At very low pressure, the thermal conductivity of the gas is near zero. When the pressure exceeds the operating range, the thermal conductivity becomes pressure independent and reaches its reference value $\kappa_{0}$. Between those pressure independent regions the Pirani vacuum sensor can be operated, excelled by the high slope in the center of the graph.

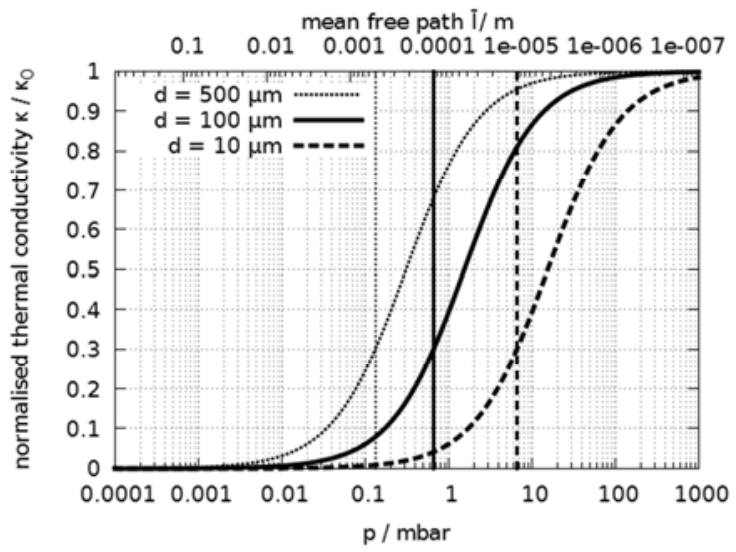

Fig. 2: Calculated thermal conductivity as a function of the pressure (lower abscissa) or mean free path (upper abscissa) for three different gap distances $d$.

In Fig. 2 the vertical lines mark the points where the mean free path equals the gap distance $d$. The operating range is always located within two decades surrounding this characteristic point.

This simple concept of pressure dependent thermal conductivity has two main disadvantages: For calculating the thermal conductivity the gap distance has to be known, so only simple geometries with constant gap distance can be calculated by eq. (2). The other disadvantage is that the real heat distribution in the system cannot be calculated. To overcome these disadvantages the following paragraph presents a method to numerically simulate an arbitrarily shaped Pirani vacuum sensor. Moreover the application of numerical methods allows the treatment of systems with convection.

To model the sensor characteristics, all mechanisms of heat transport have to be taken into account, since they all affect the characteristics and the sensitivity of the sensor.

\section{MEMS Pirani sensors}

MEMS technologies are advantageous for the fabrication of Pirani vacuum sensors. Due to the high accuracy very small gap distances are possible which allow the operation of the sensor at higher pressure levels. The small volume reduces the response time of the sensor. Both effects increase the performance and the sensitivity of the sensor.

MEMS Pirani sensors, fabricated with bulk micromachining, usually consist of an anisotropically etched silicon chip with a thin silicon nitride or oxide membrane [3], [4]. The heater is structured onto the membrane since the membrane provides small heat dissipation through its suspension. The heat sink is formed by the chip carrier or an optional silicon cover. Fig. 3 shows the general configuration of a bulkmicro-machined Pirani vacuum sensor.

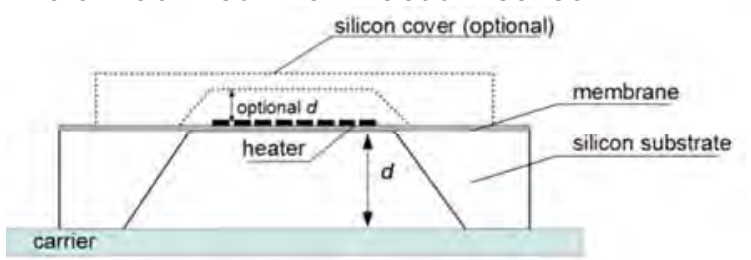

Fig. 3: MEMS Pirani sensor. A thin film heater is located on a thin membrane. The distance to the carrier forms the gab distance $d$. To operate the sensor at higher pressure, a silicon cover can optionally be mounted on top of the sensor to form a smaller gap.

\section{Numerical model of a MEMS Pirani vacuum sensor}

The numerical model of a Pirani vacuum sensor consists of two regions: the solid region, which represents the silicon chip and the membrane with heater, and the fluid region which represents the gas atmosphere around the sensor. On the interface between the solid and the fluid region (fluid-solid-interface), the boundary conditions for the temperature are exchanged to couple both regions. The dilution effects, which occur under low pressure atmosphere, are modeled by slip boundary conditions. Instead of calculating a pressure depending overall thermal conductivity $\kappa(p)$ of the gas (eq. (2)), the thermal conductivity is kept constant during the simulation process.

\section{Slip boundary conditions}

Full temperature accommodation of the gas at a fluid-solid interface requires an infinitely high frequency of collisions between the gas molecules and the boundary. This happens, when the mean free path of the gas molecules is larger than the decisive geometric dimension, e.g. the gap distance $d$. Under working conditions of a Pirani sensor, the mean free path usually does not meet this assumption. To apply the continuum model anyway, one has to introduce the von Smoluchowski boundary condition (see Fig. 4 ) [5]. Due to fewer collisions between the gas molecules and the wall the 
temperature accommodation between fluid and solid is no longer continious and a difference between the solid temperature and a fluid temperature at the interface appears.

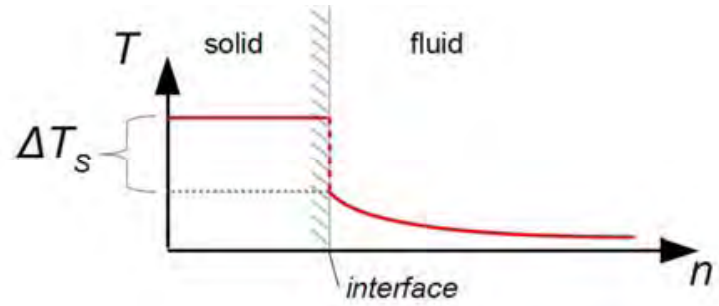

Fig. 4: Temperature slip boundary condition at the solid-fluid interface. Between the solid and the fluid region a temperature difference occurs at the boundary.

For an ideal gas the complete first order slip boundary condition for the temperature (v. Smoluchowski slip) reads as [6], [7]:

$T_{\text {fluid }}-T_{\text {solid }}=\Delta T_{S}=\frac{2-\rho_{T}}{\rho_{T}} \frac{2 k}{k+1} \frac{\bar{l}}{P r}\left(\frac{\partial T}{\partial n}\right)_{S}$

where $k$ is the heat capacity ratio, $P r$ is the Prandtl Number and $\bar{l}$ is the mean free path at the actual pressure. $\left(\frac{\partial T}{\partial n}\right)_{S}$ is the gradient in the temperature field normal to the fluid-solidinterface.

The mean free path $\bar{l}$ can be estimated as follows [8] :

$$
\bar{l}=\frac{k_{B} T}{\pi \sqrt{2} d_{m}^{2} p} \approx \frac{7 \cdot 10^{-3} \mathrm{~m} \cdot \mathrm{Pa}}{p}
$$

where $k_{B}$ is the Boltzmann constant and $d_{\mathrm{m}}$ is the diameter of the gas molecules. Comparing eq. (2) and eq. (3) shows, that the global temperature difference between the gap is transformed into a local gradient computed on the fluid-solid-interface. This makes the model independent from any geometry assumptions, like a constant distance between heater and heat sink.

All other boundary conditions of both regions are fixed values or zero gradient type.

\section{Heat radiation}

Under vacuum conditions the heat transport through radiation becomes more important, since the transport by heat conduction through the gas decreases. In a numerical simulation a radiation model can easily be implemented. The heat flux $Q$ caused by radiation can be calculated by

$$
Q=\varepsilon \sigma T_{\text {solid }}{ }^{4} A
$$

where $\varepsilon$ is the emission coefficient, $T_{\text {solid }}$ is the temperature at the solid surface, $A$ is the area of the emitting surface and $\sigma$ is the StefanBoltzmann constant. In this work the P1-Model is applied to calculate the radiative heat fluxes [9].

\section{Governing equations}

In both regions, the solid and fluid region, the heat diffusion equation has to be solved. Since we are not interested in the transient characteristic of the sensor, the time derivative can be neglected. For the fluid region the heat diffusion equation reads

$$
\nabla \cdot\left(-\kappa_{\text {fluid }} \nabla T_{\text {fluid }}\right)=0
$$

where $\kappa_{\text {fluid }}$ denotes the constant thermal conductivity of the gas. For the solid region the equation is almost the same, except for a source term which represents the Joule heating in the heater.

$$
\nabla \cdot\left(-\kappa_{\text {solid }} \nabla T_{\text {solid }}\right)=p_{\text {el }}
$$

In this equation $\kappa_{\text {solid }}$ denotes the thermal conductivity of the silicon chip and the nitride/oxide membrane. The source term $p_{\mathrm{el}}$ is the volumetric power density caused by the electrical current in the heater.

\section{Coupling of solid and fluid}

Direct matrix coupling of both regions is not feasible, since the temperature is not continuous at the fluid-solid-interface (see Fig. 4 and eq. (3)) and a radiation model has to be solved in the fluid domain. Another advantage of a sequential coupling strategy is, that the mesh need not to be conformal at the fluid-solidboundary interface. The disadvantage is a slower convergence behavior of the solver. The used coupling strategy is known as DirichletNeumann-Coupling and works as follows [10]:

1. Calculation of the temperature field in the fluid region

2. Calculation of the heat flow into the solid region. The heat flow is used as a Neumann-boundary condition for the calculation of the temperature field in the solid domain

3. Calculation of the temperature field in the solid region

4. Use the calculated temperature on the solid-fluid-interface as Dirichletboundary for the calculation of the temperature field in the fluid domain. 
This coupling strategy is expanded by the calculation of the v. Smoluchowski temperature slip and the $\mathrm{P} 1$ radiation model. The sequence diagramm of the simulation is shown in Fig. 5.

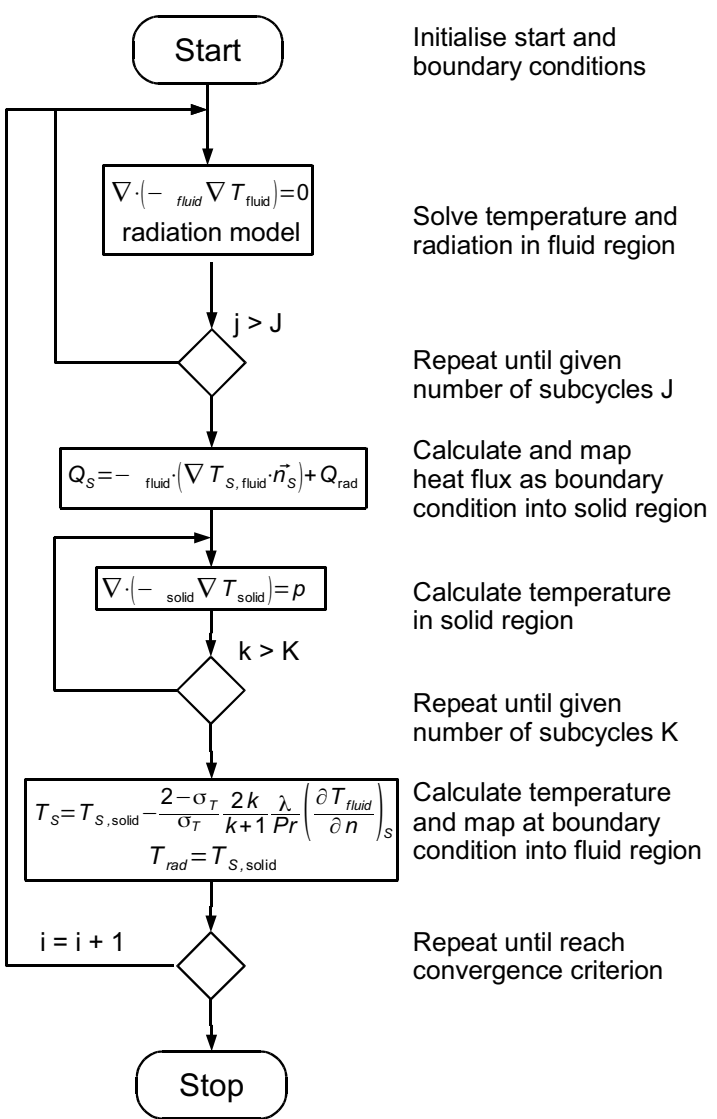

Fig. 5: Sequence diagram for the simulation procedure.

At first all start and boundary conditions are initialized. This includes the temperature fields as well as all material properties. In the second program step the temperature field in the fluid region is solved. This step is repeated for a predefined number of sub cycles $J$. Subsequently the temperature field is used to calculate the heat flux at the fluid-solid-interface. The heat flux is used as a boundary condition to calculate the temperature field in the solid region. The calculation in the solid region is repeated for a given number of sub iterations. The boundary temperature at the fluid-solidinterface and the surface normal gradient of the temperature in fluid region is used to calculate a new boundary temperature in the fluid region (slip boundary). If the desired convergence is reached the solver stops. Otherwise the whole procedure is repeated.

\section{The electrical subsystem}

Usually the heater of a Pirani vacuum sensor is operated in a Wheatstone bridge (see Fig. 6).
The Wheatstone bridge is powered by the constant voltage $U_{0}$.

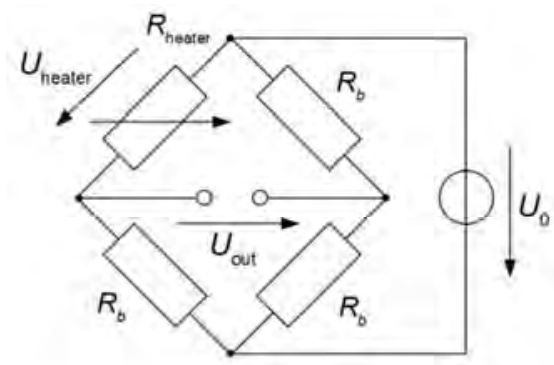

Fig. 6: Electrical configuration of a the simulated Pirani vacuum sensor. The heater is operated in a Wheatstone bridge, which is calibrated at high vacuum.

In this configuration the heater is heated by the power $P_{\mathrm{el}}=U_{\text {heater }} / R_{\text {heater }}^{2}$. Since the temperature is varies, the resistance of the heater is not constant. So the electrically generated heat source needs to be updated in every iteration step. Since the heater is operated in a Wheatstone bridge the power density in the heater reads

$$
p_{\text {el }}=\frac{P_{\text {el }}}{V}=\frac{R_{\text {heater }} U_{0}^{2}}{\left(R_{\text {heater }}+R_{b}\right)^{2} V}
$$

The resistance is calculated by the following linear formula:

$$
R_{\text {heater }}=R_{\text {heater }, 0}\left(1+\alpha\left(T-T_{0}\right)\right)
$$

where $\alpha$ denotes the thermal coefficient of the electrical resistance. These two equations are calculated after each solid region temperature calculation. To obtain the output voltage $U_{\text {out }}$ of the Wheatstone bridge, the following equation needs to be computed:

$$
U_{\text {out }}=\left(\frac{R_{0}}{R_{0}+R_{\text {heater }}}-\frac{1}{2}\right) U_{0}
$$

\section{Implementation in OpenFoam}

The described solving procedure has been implemented in OpenFOAM. OpenFOAM is a collection of $\mathrm{C}++$ libraries to solve partial differential equations in arbitrary geometry [11]. OpenFOAM uses the finite volume method (FVM) to solve differential equations. It contains programs to generate computational grids, to solve the equations and some libraries for post processing. Since it is open source, the user can adapt the solvers according to the problems to be solved. 


\section{Sensor test case}

The solver is validated with a MEMS Pirani sensor, with a similar configuration to Fig. 3, but without a silicon cover. Fig. 7 shows a 3D-CADmodel of the simulated Pirani vacuum sensor. The silicon nitride membrane has an area of $1080 \mu \mathrm{m} \times 1080 \mu \mathrm{m}$ and a thickness of $800 \mathrm{~nm}$. The thickness of the silicon chip is $500 \mu \mathrm{m}$, which represents the gap distance (see section $1)$.

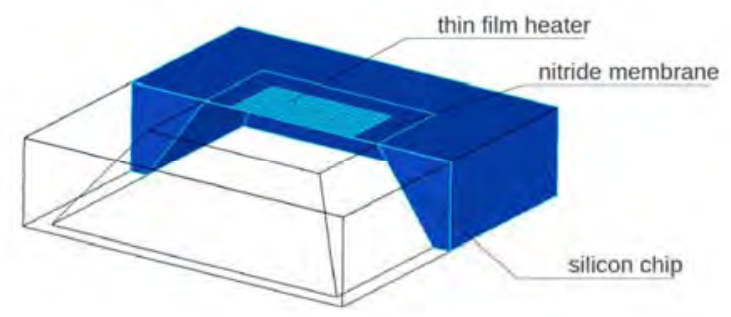

Fig. 7: 3D-Cad model of the simulated Pirani vacuum sensor with membrane area of $1080 \mu \mathrm{m} \times 1080 \mu \mathrm{m}$ and thickness of $800 \mathrm{~nm}$. The thickness of the silicon chip is $500 \mu \mathrm{m}$.

To apply the finite volume method to solve the differential equations the computational domain has to be discretized into small control volumes. Since the solver couples fluid and solid regions sequentially, both regions are meshed separately. As mentioned before, there is no need to generate conformal meshes at the fluid-solidinterface. The computational grid is depicted in Fig. 8.

The solid region is meshed with a block structured hexagonal grid. This provides the possibility to mesh thin membranes with a low number of cells. The block structured mesh provides the lowest non orthogonality which guarantees numerical stability. The fluid domain is meshed with an automatic unstructured tetragonal grid.

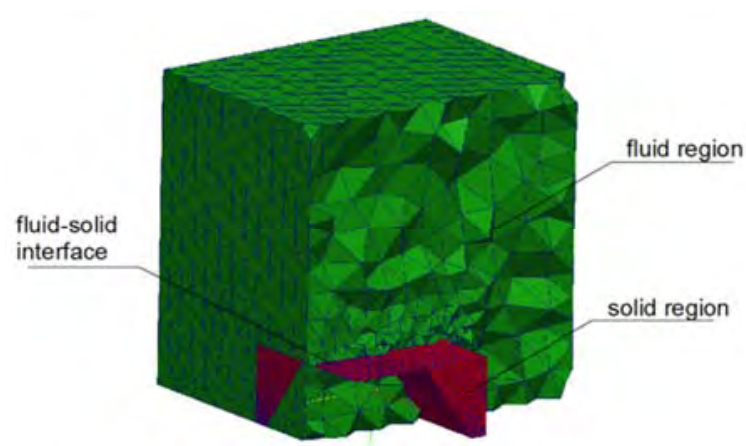

Fig. 8: Computational grid of the sensor arrangement. Green marks the fluid region, red marks the solid region. The heater is neglected for the computations. It can be seen, that the mesh is highly non conformal at the solid-fluid-interface.

Since the sensor has a quadratic footprint, symmetry planes can be used to save computational time. Two symmetry planes are intro- duced, therefore only a quarter of the sensor has to be modeled.

\section{Simulation parameters}

To start the simulation process the solver needs all of the material specific constants required by eqns. (3),(5),(6) and (7). The ambient temperature is set to $300 \mathrm{~K}$.

Tab. 1: Material properties and dimensions apllied in the simulation

\begin{tabular}{|c|c|}
\hline Material constant & value \\
\hline $\begin{array}{l}\text { Thermal conductivity of } \\
\text { silicon } \kappa_{\mathrm{Si}} / \frac{\mathrm{W}}{\mathrm{m} \mathrm{K}}\end{array}$ & 150 \\
\hline $\begin{array}{l}\text { Thermal conductivity of } \\
\text { silicon nitride } \kappa_{\mathrm{SiN}} / \frac{\mathrm{W}}{\mathrm{mK}}\end{array}$ & 2 \\
\hline $\begin{array}{l}\text { Thermal conductivity of } \\
\text { air at atmospheric pres- } \\
\text { sure } \kappa_{\text {Air }} / \frac{\mathrm{W}}{\mathrm{m} \mathrm{K}}\end{array}$ & 0,026 \\
\hline $\begin{array}{l}\text { Thermal accommodation } \\
\text { coefficient } \sigma_{T}\end{array}$ & 0.9 \\
\hline Heat capacity ratio $k$ & 1.4 \\
\hline Prandl Number $\mathrm{Pr}$ & 0.7 \\
\hline $\begin{array}{l}\text { Emissivity of the mem- } \\
\text { brane } \varepsilon_{M}\end{array}$ & 0.3 \\
\hline Membrane area $/ \mu \mathrm{m}^{2}$ & $1080 \times 1080$ \\
\hline Membrane thickness / nm & 800 \\
\hline Heated volume $/ \mu \mathrm{m}^{3}$ & $750 \times 750 \times 0,8$ \\
\hline
\end{tabular}

In order to calculate the power density according to eq. (8),(9) and (10), applied to the heater, the properties of the electrical circuit are needed. The bridge is calibrated at high vacuum. 
Tab. 2: Properties of the electrical subsystem
Material constant

Bridge resistance $R_{b} / \Omega$

1130

Resistance of the heater

$R_{\text {heater, } 0}$ at $300 \mathrm{~K} / \Omega$

Bridge input

voltage $U_{0} / \mathrm{V}$

Thermal resistance coefficient of the heater $\alpha / \frac{1}{\mathrm{~K}}$

$3.9 \cdot 10^{-3}$

\section{Simulation results}

The simulated values are compared with measured values of the same sensor setup. The output voltage characteristics are plotted in Fig. 9.

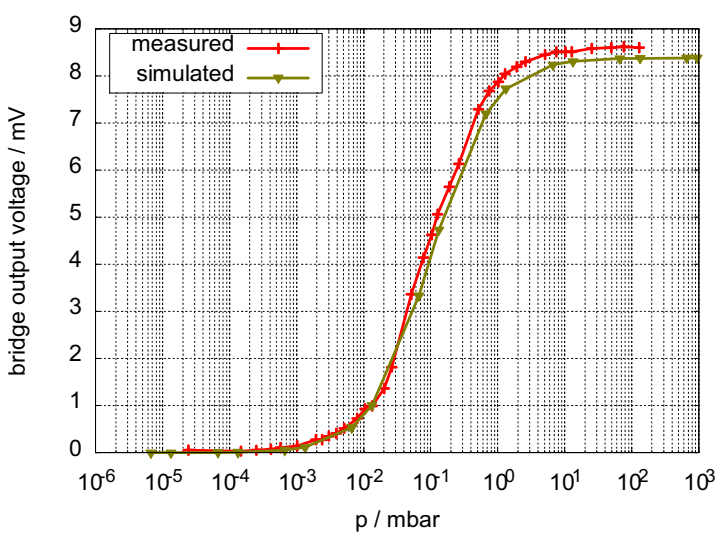

Fig. 9: Simulated and measured bridge output voltage characteristic of the Pirani vacuum sensor

It can be seen, that the simulated voltage fits well to the measured characteristic. The differences in the absolute values are due to uncertainties in applied material properties and dimensions.

To compare the pressure characteristic of the simulated and the real sensor, the normalized output characteristics are plotted in Fig. 10. It can be seen, that the normalized characteristics fit almost perfectly. This indicates that the model is suitable for the computation of heat transfer in a vacuum system.

\section{Conclusion}

The finite volume method has been applied to calculate a MEMS Pirani sensor characteristic. It has been shown, that the developed simulation algorithm can be applied to model the heat transfer in a diluted gas atmosphere. Since the simulation needs no assumption about the geometry it is not necessary to introduce a correction factor to fit the simulation results to the measured results.

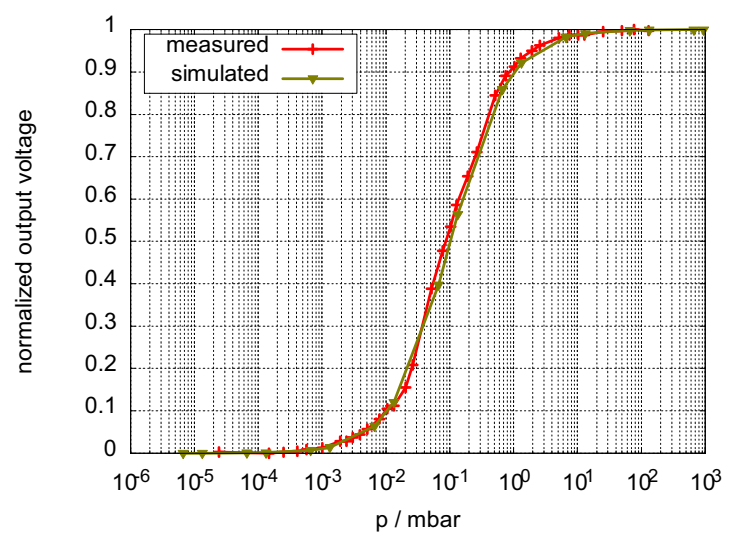

Fig. 10: Normalized pressure characteristic of the measured and the simulated values

\section{Outlook}

The developed algorithm can be applied to arbitrary geometries for the characterization of the heat transport behavior in diluted gas atmosphere. This is the case under vacuum conditions, but the same effects occur under standard pressure in MEMS, in which the dimensions are in the scale of the mean free path of gas molecules. So the algorithm offers the possibility to model heat transfer problems in a huge number of MEMS devices.

\section{Acknowledgement}

This work was supported in part by the German Federal Ministry of Education and Research (BMBF) under the grant 16SV5476.

\section{References}

[1] M. Simon, C. Dütsch, J. Schieferdecker, K. Storck, F. Völklein, und M. Grau, „MEMS Pirani type vacuum sensor with extended sensitivity range“, in 16. GMA/ITGFachtagung Sensoren und Messsysteme 2012, 2012, S. 42-47.

[2] Wei Jiang, Jilong Zhou, Xin Wang, Long Wang, und Jinwen Zhang, „Fully coupled electro-thermal simulation of a micro pirani gauge", in 2010 IEEE 5th International Conference on Nano/Micro Engineered and Molecular Systems, Xiamen, 2010, S. 1088-1091.

[3] P. Dozoretz, C. Stone, und O. Wenzel, "Shrinking the Pirani Vacuum Gauge“, SENSORS, Jan. 2005. 
[4] F. Völklein und A. Meier, „Microstructured vacuum gauges and their future perspectives“, Vacuum, Bd. 82, Nr. 4, S. 420-430, Dez. 2007.

[5] M. Gad-el-Hak, The MEMS Handbook: MEMS, design and fabrication. CRC press, 2006.

[6] L. O'Hare, T. J. Scanlon, D. R. Emerson, und J. M. Reese, „Evaluating constitutive scaling models for application to compressible microflows", International Journal of Heat and Mass Transfer, 2008.

[7] C. Shen, Rarefied gas dynamics: fundamentals, simulations and micro flows. Springer Verlag, 2005.

[8] D. Hänel, Molekulare Gasdynamik. Springer Verlag, 2004.

[9] S. S. Sazhin, E. M. Sazhina, O. FaltsiSaravelou, und P. Wild, „The P-1 model for thermal radiation transfer: advantages and limitations“, Fuel, Bd. 75, Nr. 3, S. 289294, Feb. 1996.

[10] F. Duchaine, S. Mendez, F. Nicoud, A. Corpron, V. Moureau, und T. Poinsot, „Coupling heat transfer solvers and large eddy simulations for combustion applications", in Proceedings of the Summer Program, 2008, S. 113.

[11] „OpenFOAM® Documentation“. [Online]. Available: http://www.openfoam.org/docs/. [Accessed: 29-Jan-2013]. 